\title{
Pulsation and Binarity in RZ Cas
}

\author{
E. Rodríguez ${ }^{1}$, J.M. García ${ }^{2}$, D.E. Mkrtichian ${ }^{3,4}$, V. Costa ${ }^{1}$, S.-L. Kim ${ }^{5}$, \\ M.J. López-González ${ }^{1}$, E. Hintz ${ }^{6}$, A.V. Kusakin ${ }^{7,8}$, A.Y. Gamarova ${ }^{4}$, J.W. Lee ${ }^{5}$ \\ J.-H. Youn ${ }^{5}$, E.B. Janiashvili ${ }^{9}$, R. Garrido ${ }^{1}$, A. Moya ${ }^{1}$, Y.W. Kang ${ }^{3}$ \\ ${ }^{1}$ Instituto de Astrofísica de Andalucía, CSIC, P.O. Box 3004, E-18080 Granada, Spain, E-mail:eloy@iaa.es \\ ${ }_{2}$ Departamento de Física, E.U.I.T. Industrial, UPM, Ronda de Valencia 3, E-28012 Madrid, Spain \\ ${ }^{3}$ Astrophysical Research Center for the Structure and Evolution of the Cosmos (ARCSEC), Sejong \\ University, Seoul 143-747, Korea \\ ${ }^{4}$ Astronomical Observatory, Odessa National University, Shevchenko Park, Odessa, 65014, Ukraine \\ ${ }^{5}$ Korea Astronomy Observatory, Daejeon, 305-348, Korea \\ ${ }^{6}$ Department of Physics and Astronomy, Brigham Young University, Provo, UT 84602, USA \\ 7 Sternberg State Astronomical Institute, Universitetsky prospect, 13, Moscow, 119899, Russia \\ 8 Isaak Newton Institute in Chile, Kazakhstan Branch \\ ${ }^{9}$ Abastumani Astrophysical Observatory, Abastumani, 383763, Georgia
}

\begin{abstract}
We present here a study of the Algol-type system RZ Cas from both points of view, pulsational and binary, based on a multisite photometric campaign carried out in the year 1999. Moreover, a review of the pulsational behaviour of its primary component over time (decades) is performed leading to very interesting results.
\end{abstract}

\section{Introduction}

RZ Cas is a bright Algol-type eclipsing binary system ( $\left.\mathrm{V}=6 .{ }^{m} 26, \mathrm{~A} 3 \mathrm{~V}+\mathrm{K} 0 \mathrm{IV}\right)$ where the primary component is a $\delta$ Sct-type pulsator. This system has an orbital period of $\mathrm{P}_{b}=1{ }^{d} 1953$ and eclipse depths of about $\Delta \mathrm{V} \sim 1 .^{m} 50$ and $0 .^{m} 07$ for the primary and secondary minima, respectively. The pulsational amplitude is very small in comparison $\left(\sim 0 .^{m} 015\right.$, peak to peak) with a very short main period (22 minutes) (Ohshima et al. 1998, 2001). In fact, this is the $\delta$ Sct variable with the shortest main period known (Rodríguez et al. 2000).

Despite its very low pulsational amplitude, the oscillations of RZ Cas are directly seen in the observed light curves, even during the secondary minimum and at the beginning and the end of the primary minimum, as shown in Fig. 1 for some light curves collected in the present work. Nevertheless, the history of the study of the pulsations in this system is very short. RZ Cas was discovered as eclipsing binary by Muller in 1906 and Dugan (1916) published the first complete light curve deriving the orbital elements of the system. Since then, the system has been widely observed both photometrically and spectroscopically by many authors, but the $\delta$ Sct-type variability was only recently announced by Ohshima et al. (1998).

During several decades, one very important subject of controversy in this system has been the type of primary minimum taking place, because different shapes for the light curves were found at different minima. The analyses of a number of photometric light curves carried out by different authors indicate that the primary minimum corresponds to a partial eclipse. However, partial eclipses with different shapes have been reported. Furthermore, flat bottoms in some minima were detected at other times. This has led to a number of 
different interpretations based, in all cases, on the particular characteristics of the eclipsing system: activity, starspots, etc. were deemed responsible for modifying the light curve during the primary eclipse. However, Ohshima et al. (2001) showed that the existing short-period pulsations in the primary component can be responsible for these discrepancies.

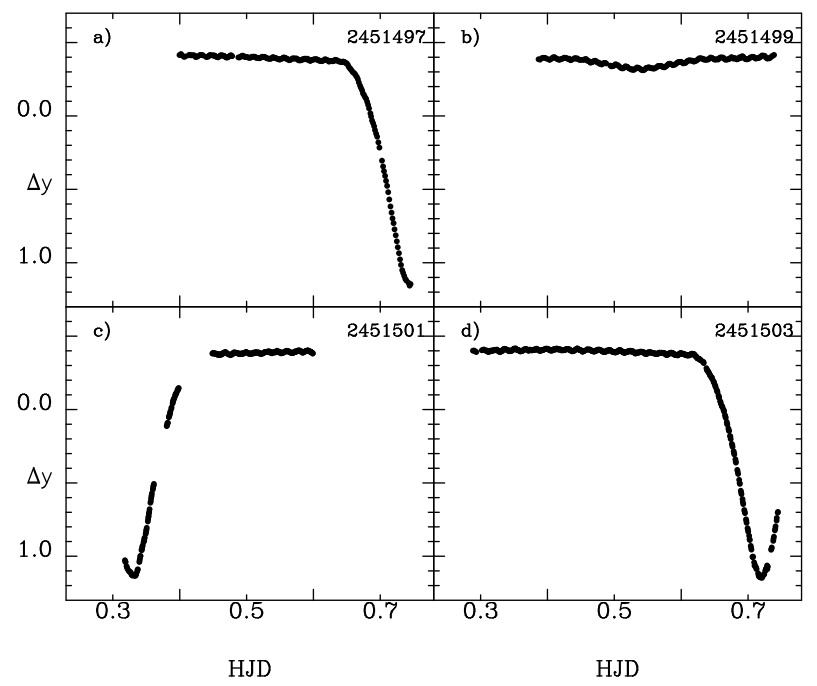

Figure 1: Some examples of nightly observed $y$ light curves of RZ Cas: a,c,d) out and during the primary eclipse and b) out and during the secondary eclipse.

\section{Observations}

In order to make a detailed study of the pulsational behaviour of RZ Cas, a coordinated multisite photometric campaign was carried out from four observatories: Sobaeksan Optical Astronomy Observatory (SOAO), South Korea; Tien-Shan Astronomical Observatory (TSAO), Kazakhstan; Sierra Nevada Observatory (SNO), Spain and Orson Pratt Observatory (OPO), USA. In total, 171 hours on 34 nights of useful data were collected over a time span of 141 days from July to December of 1999. Different detectors were used at different observatories. In particular, simultaneous observations in the four uvby filters of the Strömgren photometric system were collected at $\mathrm{SNO}$ along with a few $\mathrm{H}_{\beta}$ measurements obtained around orbital phase of 0.25 for purposes of calibration.

\section{Results}

Our main results can be summarised as follows:

a) RZ Cas is a semi-detached system where the secondary component fills its Roche lobe, with a circular orbit and with no sign of third light in the system. The appearance of the light curves reveals the presence of a hot spot on the surface of the primary as result of the mass stream coming from the secondary. There are also some indications of chromospheric activity of the secondary. 
b) The existence of pulsations of the $\delta$ Sct-type in the primary component is confirmed. It can explain the existing distortions, reported by earlier authors, in the light curves during the primary minima. This can also explain some disturbances found out-of-eclipse by other authors.

c) For data collected between 1997 (Ohshima et al. 2001) to 1999 (present work), the pulsational behaviour can be well described in a similar way: with only one frequency and constant amplitude.

d) Our results indicate that nonradial pulsation in a high radial order $(n=6)$, with $\mathrm{I}=2$, $|\mathrm{m}|=1,2$ is the most suitable mode identification. The theoretical solutions were modelled using the method of Spatial Filtration (Gamarova et al. 2003), by predicting the distortions in the pulsational light curves when the primary component is being partially hidden by the secondary.

e) The pulsation amplitude in the $u$ band is larger than in both the $b$ and $v$ bands, which is unusual among the $\delta$ Sct-type variables. Using new improvements in multicolor photometry, this can be explained as due to pulsation in a high $n$-value of a star close to the blue edge of the $\delta$ Sct region.

f) A revision of all the photometric out-of-eclipse data sets available in the literature was carried out. From this, we find: the existence of amplitude variations in the pulsations from season to season except in the interval mentioned in point c), possible multiperiodicity in some epochs and possible variations in the frequencies themselves. In fact, in a number of epochs the pulsation is not detected. If existing, it is below the photometric limit of detectability. This limit is in some cases very small $(\sim 3 \mathrm{mmag})$.

\section{References}

Dugan R.S. 1916, Contr. Princeton University Obs. No. 4

Gamarova A.Y., Mkrtichian D.E., Rodríguez E., Costa V., López-González M.J. 2003, PASPC 292, 369

Ohshima O., Narusawa S.Y., Azakawa H. et al. 1998, Inf. Bull. Var. Stars. No. 4581

Ohshima O., Narusawa S.Y., Azakawa H. et al. 2001, AJ 122, 418

Rodríguez E., López-González M.J., López de Coca P. 2000, A\&AS 144, 469 